\title{
Factores demográficos, crianza e historia de salud: vinculación con la nutrición y el desarrollo infantil ${ }^{*}$ \\ Demographic factors, rearing and health history: its relation with nutrition and child development
}

Recibido: noviembre 6 de 2009 ～Revisado: diciembre 11 de 2009 Aceptado: febrero 7 de 2010

\author{
AsSOl CORTÉS MORENO ** \\ Ana Laura Avilés Flores \\ Universidad Nacional Autónoma de México, México
}

\section{RES UMEN}

Los efectos de la desnutrición infantil sobre el desarrollo psicológico varían dependiendo de factores psicosociales. El presente estudio evaluó el impacto de variables demográficas, familiares y de crianza, sobre el estado de nutrición y el desarrollo psicológico del niño, en el periodo de alimentación complementaria. Participó una muestra de 124 parejas niño-cuidador de comunidades mexicanas, con distintos índices económicos y de riesgo nutricional. Se evaluó la antropometría y el desarrollo psicológico infantil. Se probó el poder predictivo de variables demográficas, salud familiar y prácticas de crianza. Los modelos de regresión múltiple indican mayor impacto del peso al nacer sobre el estado nutricional, seguido por variables demográficas y crianza. La crianza incrementa su peso explicativo sobre el desarrollo psicológico del niño.

Palabras clave autores

Desarrollo infantil, desnutrición, desmedro, prácticas parentales.

Palabras clave descriptores

Psicología del desarrollo, evaluación mental y psicomotora, investigación cuantitativa.

Para citar este artículo. Cortés Moreno, A. \& Avilés Flores, A. L. (2011). Factores demográficos, crianza e historia de salud: vinculación con la nutrición y el desarrollo infantil. Universitas Psychologica, 10 (3), 789-802.

* Artículo de investigación. Financiación proyecto CONACyT 41589-H.

Las autoras agradecen la invaluable colaboración de los padres y niños involucrados en el estudio, así como de las autoridades del ISSSEMyM Tlalnepantla, del ISEM Chiconaultla y del ISEM Chapa de Mota. También reconocen la participación de Miriam López, Lilia García y Gicela Flores en el trabajo de campo y la captura de datos.

** Facultad de Estudios Superiores Iztacala (FESI). E-mails: assol@servidor.unam.mx, psique_lau@ hotmail.com

\section{A B S T R A C T}

Effects of undernourishment on psychological development vary according psychosocial factors. This study assessed the impact of demographic, familiar, and rearing variables on nourishment and psychological development in children aged complementary feeding. A sample of 124 child-caregiver dyads from four different socioeconomic and nutritional index communities from México participated. Anthropometrics and child development were measured. Prediction power of demographics, rearing practices, and family health were assessed. Multiple regression models obtained illustrate a greatest impact of birth weight on nourishment condition. This factor is followed by demographic and rearing variables, which become more predictive for psychological child development.

Key words authors

Child development, malnutrition, stunting, parental practices.

Key words plus

Development psychology, mental and psychomotor assessment, quantitative research. 
El vínculo entre el estado de nutrición y el desarrollo neurológico y conductual se ha investigado ampliamente. Existe evidencia empírica suficiente para poder afirmar que cuando sobrevienen carencias de nutrimentos esenciales durante la infancia temprana y estas se prolongan, el sistema nervioso central (SNC) sufre daños que pueden dar como resultado pérdidas conductuales importantes (Pollitt et al., 1996; Sazawal et al., 1996; Wachs, 2000). Las etapas más sensibles a la desnutrición son la edad gestacional y el primer año de vida, debido a que un daño en esta fase de rápido desarrollo reduce la proliferación neuronal que no podrá ser compensada más tarde. De la misma manera, otros procesos de desarrollo del SNC, como la mielinización, continúan durante la niñez, por lo que la ocurrencia de un déficit nutricional severo tiene el potencial de modificar la tasa en que se integran estructuras neuronales relacionadas con la emergencia de diferentes habilidades cognoscitivas y conductuales (Hughes \& Bryan, 2003). Más aún, cuando la desnutrición se genera entre los 6 y 24 meses de edad, representa un gran riesgo para la sobrevivencia del niño, al producir el mayor daño a su organismo y limitar severamente el desarrollo futuro de sus capacidades.

No obstante, cuando las carencias de nutrimentos se presentan en una etapa tardía, los efectos pueden atenuarse dado que el proceso de desarrollo estructural del SNC se encuentra más avanzado y es altamente probable que el niño cuente ya con repertorios conductuales básicos, necesarios para la adquisición de procesos psicológicos más complejos. En ese momento, el contexto social que rodea al niño puede desempeñar un papel crucial. Si al niño se le brinda la estimulación y atención necesaria, los efectos de la desnutrición pueden compensarse (Drewett, Wolke, Asefa, Kaba \&Tessema, 2001).

De manera conjunta a la temporalidad de la desnutrición, otros factores actúan para determinar el tipo y alcance de sus secuelas. Entre ellos destacan la naturaleza del déficit y las condiciones sociales en las que se desenvuelve el menor.

El primer factor, el tipo de carencia, puede determinar el carácter de las consecuencias asociadas a la desnutrición, debido a que cada nutrimento tiene diferentes funciones en el organismo de acuerdo a su composición. Hay manifestaciones de una relación consistente entre la desnutrición proteico-calórica crónica y el déficit cognoscitivo, académico y conductual. La ejecución conductual puede ser particularmente sensible a una dieta pobre en productos de origen animal (Cravioto \& Arrieta, 1982; Pollitt et al., 1996). Por otra parte, carencias de diferentes micronutrimentos (hierro, zinc, calcio, fósforo, entre otros) redundan en problemas relacionados con la actividad general mostrada por el niño, como la atención y la reactividad. Así mismo, una dieta en la que estos nutrimentos se encuentran contenidos en cantidades menores a las recomendadas, contribuye a que la dimensión afectiva del comportamiento se vea alterada. Tales carencias podrían favorecer el aislamiento funcional del niño respecto de su medio físico y social (Chávez, Martínez \& Bourges, 1976; GranthamMcGregor, Powell, Walter \& Himes, 1991; Lozzoff et al., 1998; Pollitt et al., 1996).

El segundo factor, el nivel sociocultural, es igualmente crítico para entender el problema, puesto que un medio social desfavorable por sí solo puede constituir un factor de riesgo para el desarrollo del niño, sobre todo cuando este ambiente empobrecido se conjuga con un estado de nutrición deteriorado. Condiciones ligadas a la pobreza, como el nivel educativo insuficiente y la falta de recursos tanto económicos como conductuales de los padres, fungen como precipitantes de prácticas de crianza deficientes. Entre ellas destacan la omisión de la lactancia materna o bien su prolongación, la ablactación inapropiada, la falta de prevención de enfermedades infecciosas y su manejo incorrecto una vez que se presentan y, en general, la organización poco efectiva del ambiente para proporcionar estimulación de calidad que tienda a promover el desarrollo físico y psicológico del menor. Cuando las prácticas de crianza son inadecuadas, se incrementa la probabilidad de desnutrición en el infante, y cuando ya está presente, potencian sus efectos negativos (Ávila, Chávez, Shamah \& Madrigal, 1993; Black, 2003; Hernández, Barberena, Camacho \& Vera, 2003; 
Poehlmann \& Fiese, 2001). Por ejemplo, Kerr, Bogues y Kerr (1978), al comparar patrones psicosociales y personales entre mujeres con hijos que presentaban fallo en el crecimiento y mujeres con hijos cuyo desarrollo era normal, identificaron que las primeras presentaban más alteraciones frecuentes durante su vida. Sus condiciones de vivienda y la cantidad de empleos reflejaron desorganización, sus relaciones familiares y personales fueron inestables y, al relacionarse con sus hijos, algunas se mostraron hostiles o apáticas, mientras otras los ignoraban o los estimulaban en exceso, dependiendo de la situación que se presentara. Además de lo anterior, algunas características de la conducta de los cuidadores, por ejemplo, la insistencia para que el niño consuma toda la comida servida, pueden convertir la situación de alimentación en un estresor para el niño, propiciando la negativa a ingerir el alimento servido (Ramsay, 2004). Por lo anterior, la desnutrición de menores vinculada a causas no orgánicas se reconoce como un caso de maltrato infantil por negligencia de los padres, sobre todo cuando ocurre en un medio donde el acceso a los alimentos no representa un impedimento para el bienestar del niño (Kerr \& Maureen, 2000; Krugman \& Dubowitz, 2003).

En contraste, las condiciones de crianza apropiadas pueden operar como un elemento promotor del desarrollo y con ello compensar la falta de recursos económicos en la familia o la existencia de factores ambientales desfavorables. A través de prácticas de crianza sensibles y de calidad, puede asegurarse la alimentación correcta del niño para prevenir la desnutrición y, en el caso de haberla padecido, puede revertirla o minimizar las consecuencias negativas sobre el desarrollo psicológico. Las prácticas de crianza se definen por todas aquellas acciones enfocadas a cubrir las necesidades de supervivencia y desarrollo del niño, durante sus primeros meses y años de vida (Myers, 1993). La respuesta oportuna y pertinente de las madres ante las necesidades del infante, se relaciona con un nivel cognoscitivo más elevado y un desarrollo de lenguaje más acelerado durante los primeros tres años de vida (Ávila et al., 1993; Hernández et al., 2003; Pelto, 2000; Poehlmann \& Fiese, 2001). La crianza sensible puede constituirse, entonces, como un factor protector de la desnutrición infantil $y$, en todo caso, de las secuelas que conlleva sobre el desarrollo. Con base en lo anterior, podemos señalar que al igual que en otros problemas de salud, en la desnutrición y sus secuelas intervienen factores de riesgo que operan en distintos niveles de influencia, algunos de los cuales -como la pertenencia a una clase económica con índices de marginación- no son susceptibles de modificación desde la psicología; mientras que otros factores de riesgo sí pueden ser sujetos de transformación mediante intervenciones enfocadas a prevenir la desnutrición y enfermedades que la desencadenan, aminorar sus efectos cuando aparece y mejorar la calidad y expectativa de vida de los infantes.

Desde la perspectiva de la Psicología de la Salud, deben reconocerse los factores de riesgo distales, los cuales pertenecen a condiciones de vida de orden macrológico que son difíciles de cambiar y que constituyen predisponentes de problemas de salud en ciertas poblaciones, distinguiéndolos de los factores de riesgo proximales inherentes a los patrones de comportamiento, los cuales pueden modificarse para fungir como factores de protección, ante un entorno económico y cultural adverso (Sánchez-Sosa, 1998). En última instancia, la vulnerabilidad biológica de una persona depende de la estrecha relación que guardan circunstancias sociales y culturales particulares, que pueden afectar su salud, con los estilos de comportamiento y las competencias conductuales disponibles, que le permiten enfrentar dichas circunstancias (Ribes, 1990).

Concretando este punto de vista en la problemática de la desnutrición infantil, la valoración de las condiciones sociales en las que se desenvuelve el niño, el estado de salud que ha experimentado durante su vida, así como las prácticas de crianza de los cuidadores como factor regulador del estado de nutrición y desarrollo del niño, se vuelve un aspecto medular para entender y coadyuvar a la solución de este problema. En este orden de ideas, la presente investigación se realizó con la finalidad de valorar el impacto de diferentes variables demográficas, familiares, orgánicas y de crianza, 
sobre el estado de nutrición y el desarrollo psicológico del niño, en el periodo de la alimentación complementaria.

Para ello se tomaron diversas medidas en una muestra heterogénea, con el fin de obtener información de variables demográficas del medio en el que crece el infante así como variables familiares y de crianza; asimismo, se consideraron aspectos de salud, crecimiento y desarrollo del niño, incluyendo indicadores antropométricos y la aplicación de una escala desarrollo.

\section{Método}

\section{Participantes}

Se seleccionó una muestra heterogénea no probabilística de 134 niños de 9 a 24 meses de edad y sus cuidadores principales, quienes consintieron participar de manera informada. Se excluyeron los casos de niños que presentaban desnutrición derivada de algún problema metabólico o de absorción intestinal. La muestra se conformó por cuatro submuestras de acuerdo a la comunidad de origen de los participantes. Estas se distinguieron por el índice de riesgo de desnutrición para la población (Instituto Nacional de Ciencias Médicas y Nutrición Salvador Zubirán [INCMNSZ] \& Sociedad Latinoamericana de Nutrición [SLAN], 2003) y el tipo de servicios disponibles. Quedó compuesta de la siguiente forma: 1) Veintidós parejas de tres municipios conurbados de Ciudad de México, con bajo riesgo de desnutrición y con servicios urbanos completos, reclutados de manera individual por los evaluadores; 2) Cincuenta y cinco parejas reclutadas en la Clínica Familiar del ISSSEMyM Tlalnepantla, habitantes de este municipio distinguido por bajo riesgo de desnutrición, pudiendo no ser derechohabientes del Instituto; 3) Ocho parejas remitidas por el servicio de pediatría del Centro de Salud de Chiconautla, Ecatepec perteneciente al Instituto de Salud del Estado de México (ISEM). Este municipio está caracterizado con bajo riesgo de desnutrición, pero el centro de salud se encuentra enclavado en una zona con altos índices de marginación y 4) Cuarenta y nueve parejas contactadas a través del Centro de Salud (ISEM) de Chapa de Mota, municipio con muy alto riesgo de desnutrición.

\section{Aparatos y materiales}

Un infantómetro de aluminio Seca Modelo 207, con exactitud de un milímetro; una báscula electrónica marca Tanita 1582 con precisión de 20 g; escalas Bayley de Desarrollo Infantil (BSID), versión española (1997), y formatos para las diferentes medidas.

\section{Medidas}

\section{Indicadores antropométricos}

Las medidas de peso y longitud supina se efectuaron de acuerdo a la técnica recomendada por la Organización Mundial de la Salud (World Health Organization [WHO], 1995). Los datos obtenidos se procesaron para obtener las puntuaciones z con el programa ANTHRO 2005 (WHO, 2006), que emplea una nueva referencia poblacional, comprendiendo sesgos regionales y tendencias seculares actualizadas.

\section{Alimentación}

Se preguntó a la madre sobre los alimentos proporcionados al niño, durante las 24 horas previas a la entrevista. Se incluyó información sobre el número de comidas principales y complementarias, cantidad de alimentos, composición y modo de preparación. La información se transformó en un índice de adecuación de consumo de alimentos (IACA), una versión modificada del índice desarrollado por Ruel y Menon (2002). El puntaje (12 máximo) se asignó de acuerdo a los aspectos contenidos en la Tabla 1.

\section{Desarrollo infantil}

Se evaluó con las escalas de Desarrollo de Bayley (BSID) que proporcionan una base de evaluación a través de dos componentes: a) Índice de Desarrollo 
TABLA 1

Valores asignados a la alimentación de acuerdo con Ruel y Menon

\begin{tabular}{|c|c|c|}
\hline Variables & $9-12$ meses & $12-36$ meses \\
\hline $\begin{array}{l}\text { Lactancia } \\
\text { Uso de mamila }\end{array}$ & $\begin{array}{l}\text { No }=0 ; S i ́=2 \\
N_{0}=1 ; S i ́=0\end{array}$ & $\begin{array}{l}N_{0}=0 ; S i ́=1 \\
N_{0}=1 ; S i ́=0\end{array}$ \\
\hline Diversidad de la dieta & $\begin{array}{l}\text { Suma de (cereales y tubérculos }+ \text { frutas }+ \\
\text { verduras }+ \text { lácteos }+ \text { carnes }) \\
0=0 \\
1-3=1 \\
4 \text { o más }=2\end{array}$ & $\begin{array}{l}\text { Suma de (cereales y tubérculos }+ \text { frutas }+ \\
\text { verduras }+ \text { lácteos }+ \text { carnes }) \\
0=0 \\
1-3=1 \\
4 \text { o más }=2\end{array}$ \\
\hline $\begin{array}{l}\text { Frecuencia de alimentos } \\
\text { (durante } 7 \text { días) }\end{array}$ & $\begin{array}{l}\text { Carne } \\
0 \text { veces }=0,1-3 \text { veces }=1, \\
4 \text { veces }=2 \\
\text { Cereales y tubérculos } \\
0-3 \text { veces }=0,4+=1 \\
\text { Frutas y verduras } \\
0 \text { veces }=0 ; 1-3 \text { veces }=1 ; 4 \text { o más veces }=2\end{array}$ & $\begin{array}{l}\text { Carne } \\
0 \text { veces }=0 ; 1-3 \text { veces }=1 ; \\
4 \text { veces }=2 \\
\text { Cereales y tubérculos } \\
0 \text { veces }=0,1-3=1,4+=2 \\
\text { Frutas y verduras } \\
0 \text { veces }=0, ; 1-3 \text { veces }=1 ; 4 \text { o más veces }=2\end{array}$ \\
\hline $\begin{array}{l}\text { Cantidad de comidas } \\
\text { efectuadas en un día }\end{array}$ & $\begin{array}{l}0 \text { veces } / \text { día }=0 \\
1-2 \text { veces } / \text { día }=1 \\
3 \text { veces } / \text { día }=2\end{array}$ & $\begin{array}{l}0-1 \text { veces } / \text { día }=0 \\
2-3 \text { veces } / \text { día }=1 \\
4 \text { veces } / \text { día }=2\end{array}$ \\
\hline Puntuación Total & 12 puntos & 12 puntos \\
\hline
\end{tabular}

Nota. Las comidas son consideradas sí éstas son sólidas y semisólidas omitiendo dulces y frituras industrializadas.

Fuente: elaboración propia.

Mental (IDM): da cuenta de la agudeza sensoperceptiva, discriminación y capacidad de respuesta a estímulos, memoria y resolución de problemas, primeras vocalizaciones, así como clasificación y generalización tempranas y b) Índice de Desarrollo Psicomotriz (IDP) que proporciona información sobre el grado de control corporal, la coordinación de músculos grandes y la coordinación fina de manos y dedos.

\section{Factores sociodemográficos e historia de salud}

Se diseñó un cuestionario con los principales datos demográficos que incluía aspectos de conformación familiar, nivel educativo familiar y características de la vivienda. Se administró también un cuestionario sobre prácticas de salud en la familia e historia de salud del niño, donde se indagó sobre las condiciones perinatales del niño, antecedentes de lactancia y enfermedades recurrentes, así mismo el cuestionario incluyó prácticas de higiene y de prevención y tratamiento de enfermedades.

\section{Crianza}

Se aplicó el Cuestionario de Práctica Responsiva y Estimulación (CuPRE) que consta de 23 reactivos con cinco opciones de respuesta, diseñado como una herramienta para detectar la sensibilidad y efectividad del cuidador para satisfacer las necesidades básicas -físicas y psicológicas- del niño. El instrumento muestra buena consistencia interna $($ alfa $=0.83)$. El CuPRE valora la práctica de crianza a través de cinco factores: estimulación a partir de juego, práctica responsiva, promoción de competencias, atención planeada y disposición para atender las demandas del niño (Cortés, Romero \& Flores, 2006).

\section{Procedimiento}

Una vez informados los cuidadores sobre la finalidad del estudio y las actividades en los que ellos y sus niños participarían, cada pareja fue evaluada por dos observadores, previamente entrenados en 
la utilización de los instrumentos. En primera instancia se tomaron las medidas de peso y longitud del niño y, posteriormente, los observadores aplicaron las escalas de desarrollo. Después de evaluar al menor, se entrevistó al cuidador aplicando los cuestionarios sobre aspectos de salud, demográficos, alimentación y el CuPRE.

\section{Análisis de resultados}

Se realizaron los análisis de distribución de peso y longitud para la edad. Para conocer la asociación entre el estado de nutrición, las distintas variables demográficas, de crianza e historia de salud y los puntajes en las pruebas de desarrollo infantil, se obtuvieron distintos modelos de regresión múltiple por etapas, mediante el método de mínimos cuadrados. Todos los análisis se realizaron empleando el paquete estadístico JMP, versión 5.1.

\section{Resultados}

\section{Indicadores antropométricos}

La determinación del grado del estado de nutrición se basó en la clasificación empleada en la Norma Oficial Mexicana para vigilancia del desarrollo estado de salud del niño (Secretaría de Salud, 1999). En ella se establece longitud y peso normales a una desviación estándar de la puntuación $Z$ alrededor de la media: desnutrición leve, moderada y grave con una, dos y tres desviaciones típicas por debajo de la media, respectivamente.

De la muestra total el $53.3 \%$ de los niños presenta una longitud normal para la talla, el resto de los niños presenta desmedro en diferentes grados: $27.8 \%$, leve; $18.7 \%$, moderado y $3 \%$, grave. Con respecto al indicador de peso para la edad, se encontró una proporción mayor $(82.7$ \%) de niños dentro de los parámetros normales, mientras que un $14.9 \%$ presenta insuficiencia ponderal leve, un 1.5 moderada, y grave solamente el $0.7 \%$ de los niños. La Tabla 2 muestra la distribución de los niños de acuerdo al estado de nutrición en cada una de las submuestras. Se encontraron diferencias significativas relacionadas con el origen, tanto en la longitud $\left(F_{(3,129)}=2.85, p<0.05\right)$ como para el peso $\left(F_{(3,130)}=3.8, p<0.05\right)$. De estos indicadores, únicamente el peso se ve afectado por el género de los niños $(t=-1.97, p=0.05)$, ya que los varones presentan pesos más bajos que las niñas; no hubo diferencias significativas en la longitud entre niños $y$ niñas $(t=-0.02, p>0.05)$.

\section{Prueba de desarrollo}

Para la calificación de las escalas de Desarrollo de Bayley, cuyo resultado se expresa en puntuaciones

TABLA 2

Distribución de longitud y peso para la edad de acuerdo al origen (submuestra)

\begin{tabular}{|c|c|c|c|c|c|c|c|c|}
\hline & \multicolumn{8}{|c|}{ Origen } \\
\hline & \multicolumn{2}{|c|}{1} & \multicolumn{2}{|c|}{2} & \multicolumn{2}{|c|}{3} & \multicolumn{2}{|c|}{4} \\
\hline & $\mathrm{N}$ & $\%$ & $\mathrm{~N}$ & $\%$ & $\mathrm{~N}$ & $\%$ & $\mathrm{~N}$ & $\%$ \\
\hline \multicolumn{9}{|l|}{ LONGITUD } \\
\hline Normal & 13 & 59.1 & 34 & 63.0 & 3 & 37.5 & 17 & 34.7 \\
\hline Desmedro leve & 2 & 09.1 & 14 & 26.0 & 4 & 12.5 & 17 & 34.7 \\
\hline Desmedro moderado & 6 & 27.3 & 4 & 07.4 & 1 & 50.0 & 14 & 28.6 \\
\hline Desmedro grave & 1 & 04.5 & 2 & 03.6 & 0 & 00.0 & 1 & 02.0 \\
\hline \multicolumn{9}{|l|}{ PESO } \\
\hline Normal & 20 & 90.9 & 47 & 85.4 & 7 & 87.5 & 37 & 75.5 \\
\hline Insuficiencia leve & 2 & 09.1 & 8 & 14.6 & 0 & 00.0 & 10 & 20.4 \\
\hline Insuficiencia moderada & 0 & 00.0 & 0 & 00.0 & 1 & 12.5 & 1 & 02.0 \\
\hline Insuficiencia grave & 0 & 00.0 & 0 & 00.0 & 0 & 00.0 & 1 & 02.0 \\
\hline
\end{tabular}

Fuente: elaboración propia. 
típicas, se consideran casos de puntuación menor a la esperada aquellos que están por debajo de una desviación estándar (puntaje igual o menor a 84), la puntuación esperada para la edad es alrededor de una desviación estándar respecto de la media (de 85 a 116) y hasta dos puntuaciones por encima de ésta (de 117 a 132). Se encontró que los puntajes promedio de los tres grupos se ubican dentro de los valores esperados en ambas pruebas, aunque en todos los grupos hubo casos con puntajes por debajo de lo esperado (Tabla 3). No se encontraron diferencias atribuibles al grupo de origen $\left(F_{(3,130)}=\right.$ $0.55, p>0.05$ para IDM; $F_{(3,130)}=0.25, p>0.05$ para IDP), ni al género de los niños $(t=-1.1597$, $p>0.05$ para IDM; $t=-0.50, p>0.05$ para IDP).

TABLA 3

Puntajes de las Escalas Mental y Psicomotora de Bayley por origen (submuestra)

\begin{tabular}{cccccccc}
\hline & \multicolumn{3}{c}{ IDM } & \multicolumn{3}{c}{ IDP } \\
\cline { 2 - 7 } \\
& Media & DE & $\begin{array}{c}\text { Min- } \\
\text { Max }\end{array}$ & Media & DE & $\begin{array}{c}\text { Min- } \\
\text { Max }\end{array}$ \\
\hline 1 & 100.18 & 13.00 & $76-127$ & 105.80 & 18.11 & $64-133$ \\
2 & 95.81 & 14.00 & $64-122$ & 100.37 & 17.48 & $52-132$ \\
3 & 96.37 & 18.47 & $71-122$ & 91.25 & 24.72 & $50-127$ \\
4 & 97.67 & 11.84 & $72-123$ & 100.12 & 15.68 & $71-128$ \\
\hline
\end{tabular}

Fuente: elaboración propia.

\section{Factores demográficos, historia de salud} y crianza asociados a la antropometría

Para obtener modelos de regresión lineal múltiple que expliquen la participación de variables de distinto orden como predictores de la longitud y el peso para la edad, medidas en puntuaciones $z$, se probaron los siguientes factores: a) Historia de Salud: peso al nacer, gestación (a término o prematuro), dificultad perinatal, salud regular y apetito; b) Crianza: lactancia, puntaje del IACA, puntaje CuPRE y c) Demográficos y familiares: comunidad de origen, cuidador, tipo de familia, orden de nacimiento, edad y género del niño, escolaridad de los padres, edad de la madre y servicios de salud.

\section{Longitud para la edad}

El mejor modelo obtenido para el indicador longitud para la edad, explica el $31 \%$ de la varianza $\left(F_{(8,124)}=6.97, p<0.0001\right)$. Los predictores pertenecen básicamente a las dimensiones demográficas y de historia de salud (Tabla 4). De las variables demográficas que ingresaron al modelo, la edad y escolaridad del padre mostraron efectos positivos -a mayor valor, tallas más grandes-; también se encontró que los niños que viven en familias extendidas y en comunidades con mejores indicadores de vida (origen 1 y 2), se aproximaron más a los parámetros normales de longitud. En contraste, el orden de nacimiento tiene un efecto negativo: cuando el niño rebasa el tercer lugar entre los hermanos, las puntuaciones $Z$ de longitud para la edad disminuyen.

Entre las variables de historia de salud del niño las únicas que participan en la explicación de la varianza son el peso al nacer y el tiempo de gestación, destacándose la primera como la de mayor importancia de todas las variables incluidas en el

\section{TABLA 4}

Relación entre la longitud para la edad (puntuación z) y variables demográficas, historia de salud y prácticas de crianza

\begin{tabular}{lcc}
\hline \multicolumn{1}{c}{ Variables } & $\beta^{\S}$ & Prob $>|t|$ \\
\hline Constante & $-1.72(0.87)$ & 0.05 \\
Origen $(4 \& 3-2 \& 1)$ & $-0.25(0.12)$ & 0.04 \\
Edad & $0.06(0.03)$ & 0.04 \\
Escolaridad del padre & $0.05(0.03)$ & 0.05 \\
Tipo de familia & $-0.24(0.11)$ & 0.04 \\
Orden de nacimiento $(3 \& 4-$ & $-0.42(0.20)$ & 0.04 \\
5\&6\&7\&8) & $0.69(0.18)$ & $<0.01$ \\
Peso nacer & $-0.24(0.11)$ & 0.04 \\
Gestación & $-0.59(0.15)$ & $<0.01$ \\
Factor1 &
\end{tabular}

Nota. R2 del modelo $=31 \%($ R2 ajustada $=27 \%), n=133$ $\S$ Coeficiente de regresión, efecto en puntuaciones z (error estándar).

Fuente: elaboración propia. 
modelo con un efecto negativo. El tiempo de gestación muestra un efecto similar, ya que los niños pretérmino muestran talla más bajas que los niños nacidos a término.

Por último, de las variables relacionadas con la crianza del niño se retuvo en el modelo solamente el Factor 1 del CuPRE, referido a Estimulación a partir del Juego. Esta variable presenta una relación negativa con la longitud del niño: puntajes más altos en este factor están asociados con tallas bajas.

\section{Peso para la edad}

Para explicar la variable de peso para la edad, el mejor modelo explicó el 36\% de la varianza $\left(F_{(7,126)}\right.$ $=9.1799, p<0.0001)$. Las dimensiones que cobran mayor importancia corresponden a la historia de salud del niño y las condiciones demográficas (Tabla 5).

Entre las variables demográficas que destacan se encuentran el origen, el orden de nacimiento y la edad, variables cuyos efectos y pesos son similares

\section{TABLA 5}

Relación entre el peso para la edad (puntuación z) y variables demográficas, historia de salud y prácticas de crianza

\begin{tabular}{lcc}
\hline \multicolumn{1}{c}{ Variables } & $\beta^{\S}$ & Prob $>|t|$ \\
\hline Constante & $-3.20(0.61)$ & $<0.01$ \\
Origen $(4 \& 3-2 \& 1)$ & $-0.34(0.08)$ & $<0.01$ \\
Edad & $0.05(0.02)$ & 0.01 \\
Género & $-0.26(0.08)$ & $<0.01$ \\
Orden de nacimiento (3\&4- & $-0.45(0.15)$ & $<0.01$ \\
5\&6\&7\&8) & $0.57(0.12)$ & $<.0 .01$ \\
Peso nacer & $-0.31(0.11)$ & $<0.01$ \\
Apetito(bueno-regular \& malo) & $0.09(0.04)$ & 0.11 \\
Número comidas principales & $0.09(0.04)$ & 0.03 \\
\hline IACA & 0.09 \\
\hline
\end{tabular}

Nota. R2 del modelo $=37 \%($ R2 ajustada $=33 \%), n=133$ ${ }^{\S}$ Coeficiente de regresión, efecto en puntuaciones z (error estándar)

Fuente: elaboración propia. a los observados en el modelo de talla para la edad. La variable género interviene a favor de las niñas quienes presentan puntuaciones $z$ de peso para la edad más altas que los niños.

Como en el caso de la longitud, el peso al nacer es la variable con más valor de predicción para explicar la varianza, encontrándose una relación positiva entre este factor y el peso en los primeros dos años de vida. Otra variable de historia de salud es el reporte de buen apetito relacionado con mejores indicadores de peso. Solamente, se incluyen en el modelo dos variables pertenecientes a la dimensión de crianza: el número de comidas principales y los valores del índice de adecuación de consumo de alimentos (IACA), ambas con una relación positiva con la variable dependiente. No obstante lo anterior, el número de comidas principales no alcanza un nivel de significancia estadística aceptable.

\section{Factores demográficos, historia de salud, antropometría y crianza asociados al desarrollo del niño}

Para desarrollar los modelos predictores de la varianza de los puntajes obtenidos en las pruebas psicomotora y mental, se seleccionaron las mismas variables empleadas para los modelos de longitud y peso, excepto apetito. En la dimensión de salud del niño se incluyeron además las puntuaciones $Z$ de longitud. No se incorporó al análisis el peso para la edad para evitar colinealidad en el modelo, ya que ambos indicadores tienen una alta correlación entre sí.

\section{Índice de Desarrollo Mental}

El mejor modelo para explicar la varianza del puntaje arrojado por la prueba de desarrollo mental (IDM), se forma con la participación de variables relativas a la historia de salud del niño y de la familia, variables demográficas, y variables relacionadas con las prácticas de crianza (Tabla 6). Este explica el $25.9 \%$ de la varianza con un valor de $F_{(7,124)}=5.93$, resultando altamente significativo $(p<0.0001)$. 
Los aspectos relacionados con la historia de salud del niño juegan un papel de primer orden. Dentro de estos, se encontró una relación positiva entre los factores compuestos por los meses de gestación y la talla para la edad con el puntaje en la prueba mental; también se encontró que el reporte de una buena salud familiar está asociado a mayores puntajes en esta evaluación. De las variables demográficas solamente la edad del niño participa con un efecto negativo, en cambio, las variables relacionadas con las prácticas de crianza muestran efectos variados: mayor diversidad en la alimentación así como puntajes más altos en el Factor 3 del cuestionario, relativo a la promoción de competencias, están asociados a puntajes más altos en el IDM; no obstante, calificaciones más altas del Factor 2 del mismo instrumento, que valora la sensibilidad del cuidador, se asociaron con puntuaciones menores de la prueba de desarrollo mental, aunque esta relación no resultó estadísticamente significativa. Cabe aclarar que esta variable se retuvo por su contribución a la explicación de la varianza.

TABLA 6

Relación entre el IDM y variables demográficas, historia de salud y prácticas de crianza

\begin{tabular}{lcc}
\hline \multicolumn{1}{c}{ Variables } & $\beta^{\S}$ & Prob $>|t|$ \\
\hline Constante & $91.80(9.53)$ & $<0.01$ \\
Edad & $-0.74(0.29)$ & 0.01 \\
Gestación & $3.86(1.27)$ & $<0.01$ \\
Longitud & $2.32(0.75)$ & $<0.01$ \\
Salud familiar ( buena-regu- & $10.67(2.75)$ & $<0.01$ \\
lar \& mala) & $-3.49(1.88)$ & 0.06 \\
Factor2 & $3.89(1.43)$ & $<0.01$ \\
Factor3 & $4.78(2.19)$ & 0.03 \\
$\begin{array}{l}\text { Diversidad } \\
\text { Nota. R2 del modelo = 25.9\% (R2 ajustada }=21 \%), n= \\
\text { 132 }\end{array}$ & \\
$\begin{array}{l}\text { Coeficiente de regresión, efecto en puntuaciones del IDM } \\
\text { (error estándar). }\end{array}$ & \\
Fuente: elaboración propia. &
\end{tabular}

\section{Índice de Desarrollo Psicomotor}

El modelo con más poder predictivo tiene un valor de $F_{(8,123)}=6.51, p<0.0001$ y explica el $30 \%$ de la varianza, e incluye factores de las tres dimensiones evaluadas (Tabla 7).

Los factores relativos a la historia de salud del niño que participan en el modelo, con una relación positiva con la variable dependiente, son los meses de gestación y la longitud del niño. En el mismo sentido, cuando los cuidadores indican que la familia del niño goza de buena salud, los puntajes de la prueba psicomotora son más elevados que cuando indican una salud familiar regular o mala.

Las variables demográficas retenidas fueron el orden de nacimiento y el servicio médico. El primero muestra efectos negativos sobre la calificación de la prueba psicomotora, a partir del segundo lugar entre los hermanos, mientras que el servicio médico indica que cuando la familia disfruta de un servicio formal, público o privado, el niño puntúa más alto, no así cuando la familia tiene que recurrir a un dispensario.

\section{TABLA 7}

Relación entre el IDP y variables demográficas, historia de salud y prácticas de crianza

\begin{tabular}{lcc}
\hline \multicolumn{1}{c}{ Variables } & \multicolumn{1}{c}{$\beta^{\S}$} & Prob $>|t|$ \\
\hline Constante & $102.23(16.24)$ & $<0.01$ \\
Gestación & $5.32(1.68)$ & $<0.01$ \\
Longitud & $3.31(0.96)$ & $<0.01$ \\
Orden nacimiento (1- & $-4.11(1.54)$ & $<0.01$ \\
$2 \& 3 \& 4 \& 5 \& 6 \& 7 \& 8)$ & & \\
$\begin{array}{l}\text { Serv. Médico (privado \& } \\
\text { público-otro \& dispensario) }\end{array}$ & $-10.58(4.61)$ & 0.02 \\
$\begin{array}{l}\text { Salud familiar (buena- } \\
\text { regular \&mala) }\end{array}$ & $-9.86(3.56)$ & $<0.01$ \\
Factor 4 & $-6.54(2.89)$ & 0.02 \\
Factor 5 & $3.70(1.63)$ & 0.02 \\
Principales & $6.72(1.67)$ & $<0.01$ \\
\hline
\end{tabular}

Nota. R2 del modelo $=30 \%($ R2 ajustada $=25.2 \%), n=132$

$\S$ Coeficiente de regresión, efecto en puntuaciones del IDP (error estándar).

Fuente: elaboración propia. 
En cuanto a las variables de crianza, son dos factores del CuPRE los que contribuyen a la obtención de puntajes en el IDP. El Factor 4 indica que cuanto más programados son los cuidados hacia el niño, éste puntúa más bajo en el IDP; no así el Factor 5, relativo a la disposición del cuidador para atender al niño cuando lo requiera, que mantiene una relación positiva con el puntaje. Por último, el número de comidas principales que proporciona el cuidador, está relacionado también de manera positiva positivamente con este índice.

\section{Discusión}

El presente estudio tuvo como objetivo evaluar la relación entre diferentes factores y la condición nutricia, así como sus resultantes en el desarrollo infantil, en una muestra constituida por menores entre 9 y 24 meses de edad. A partir de los resultados obtenidos, puede decirse que distintos factores de orden demográfico, de crianza e historia de salud, configuran arreglos particulares que actúan sobre el crecimiento físico y el desarrollo psicológico. Esto es, las variables de cada dimensión tienen efectos diferenciales en los indicadores antropométricos y los puntajes obtenidos en las pruebas de desarrollo.

Como se mostró en los resultados, despunta el peso al nacer como la variable con mayor poder de predicción para las puntuaciones $z$ de longitud y peso para la edad; así mismo, la prematurez se manifestó como un factor importante para el indicador de talla, tal como se ha encontrado en otros estudios donde el peso al nacer es el factor de riesgo con mayor peso para la estatura y peso para la edad (Chopra, 2002; Velázquez, Larramendy \& Rubio, 1998). Estos hallazgos muestran consistencia con los estudios que indican el efecto a largo plazo de la desnutrición en etapas tempranas sobre el crecimiento del niño (Prentice et al., 2008; Walker et al,. 2007) e indican el alcance de la historia de salud y la importancia de los cuidados maternos durante la etapa de gestación. Una pobre condición nutricia de nacimiento señala una desnutrición gestacional y su impacto sobre el crecimiento perdura al menos hasta las edades comprendidas en este estudio.
De los factores demográficos fueron el orden de nacimiento y el origen los que afectaron ambos indicadores antropométricos. Parkinson, Wright y Drewett (2004) al realizar una comparación de niños con fallo en el crecimiento con su pares eutróficos, no encontraron diferencias en las configuración familiar, excepto en el orden de nacimiento. Se sabe también que la procedencia de regiones rurales y/o de zonas con altos índices de marginación, es un factor de riesgo para padecer problemas de bajo peso o desmedro, dado que las condiciones precarias de los servicios sanitarios, de vivienda y de vida familiar de dichas zonas, limitan la alimentación y comprometen la salud de sus habitantes (Chopra, 2002; Myers, 1993; Peña \& Bacallao, 2002). La educación del padre, que mostró una relación positiva con la talla para la edad, se constituyó como un predictor importante para el estado de nutrición, coincidiendo con un estudio reciente donde un nivel educativo más alto en el padre resultó ser un poderoso factor de protección, incluso por arriba del nivel de escolaridad de la madre, cuando los niños viven en zonas rurales (Seemba et al., 2008). Estos autores atribuyen la fuerza predictiva de la educación paterna a su posible asociación con mayores ingresos familiares por un lado y, por otro, con la participación en la toma de decisiones junto con la madre sobre los cuidados que llevan a conservar la salud del niño. Por otra parte, la asociación encontrada entre familia extendida y mejor condición nutricia, se suma a la evidencia que indica que las familias extendidas exhiben mayores recursos conductuales para el cuidado del infante que las nucleares (Lamontagne, Engle \& Zeitlin, 1997), ya que comparten las responsabilidades de crianza entre los integrantes, jóvenes y adultos, y establecen así una experiencia social más enriquecedora para el desarrollo infantil.

Las prácticas de crianza generales parecen tener poco peso explicativo en los indicadores antropométricos. Sin embargo, las prácticas focalizadas hacia la alimentación tuvieron impacto en el peso para la edad, a través del número de comidas principales y el puntaje total del IACA. Es de esperarse que niños que reciben más comidas completas du- 
rante el día, desarrollen más peso corporal y, de la misma forma, quienes reciben una dieta correcta y acorde a su edad se acerquen más a los parámetros de normalidad. Hay que recordar que el puntaje del IACA refleja varios aspectos de la práctica alimentaria, entre ellos la frecuencia de alimentación, la lactancia, uso de biberón, y la cantidad y variedad de alimentos consumidos. Relacionado con lo anterior, los datos señalan que cuando el adulto percibe al niño con más apetito, alcanza mejor peso; estos resultados pueden señalar un encadenamiento entre la percepción del adulto acerca de las necesidades del niño y su respuesta oportuna para proporcionar un mayor número de comidas y una dieta más adecuada a su edad, como describen algunos autores los mecanismos a través de los cuales la práctica de crianza responsiva, donde existe una sincronía entre el infante y su cuidador, promueve una mejor condición nutricia (Ramsay, 2004; Wright \& Birks, 2000).

Ahora bien, cuando se analizan las variables predictoras de los índices de desarrollo, desaparece el peso explicativo de la comunidad de procedencia y adquieren importancia variables relativas a las condiciones familiares, la condición biológica del niño y las prácticas de crianza.

Se ha reiterado que mejores condiciones sociales redundan en un mayor bienestar familiar y expectativas más amplias para el despliegue de recursos conductuales para el niño (véanse, por ejemplo, Lima et al., 2004). Los resultados de este estudio muestran la importancia de contar con un servicio médico formal, sea público o privado, así como de la condición de salud de la familia, como variables que impactan positivamente las habilidades mostradas por el menor. Este impacto puede actuar al menos a través de dos vías: una buena condición de salud en una familia refleja un conjunto de cuidados y prácticas adecuadas entre sus miembros, prácticas que pueden incluir el proporcionar cuidados que promueven la salud y desarrollo del niño; la otra forma de impacto es a través de un ambiente familiar libre del estrés que generan las enfermedades cuando están presentes, lo que propicia formas de interacción más favorecedoras para el desarrollo.
Los resultados también apuntan hacia la corroboración de los hallazgos reportados por la literatura antecedente, en cuanto a que la historia de nutrición afecta el desempeño en pruebas de desarrollo. Hay que recordar que la baja longitud para la edad o desmedro, es un indicador de desnutrición crónica y resultó con una fuerza asociativa importante para ambos indicadores de desarrollo (IDM e IDP). Es posible que además de episodios de desnutrición acumulados, los niños con desmedro padecieran también anemia. Pese a que no fue posible medir las concentraciones séricas de hemoglobina, existe evidencia que en niños menores de dos años la longitud para la edad es el mejor predictor de concentraciones séricas de hemoglobina, seguido por el nivel socioeconómico (Villalpando, Shamah, Ramírez, Mejía \& Rivera, 2003). Entonces, cabe la posibilidad de que la anemia también esté jugando un papel importante en estos resultados, afectando el nivel de desarrollo psicomotor y el desempeño en las pruebas de agudeza sensoperceptiva, discriminación, vocalizaciones, memoria y generalización de la escala mental de Bayley, como lo indican varios estudios al respecto (Black, 2003; De Andraca, Salas, De la Parra \& González, 1993; Lozzoff et al., 1998; Walker et al., 2007).

De la historia de salud del niño, también la condición de nacimiento tomó un lugar dentro del conjunto de variables asociadas al desarrollo psicológico, dado que el haber nacido prematuro se asoció con mejores puntajes en las escalas aplicadas. ¿Cómo puede afectar la prematurez el desarrollo en el sentido que muestran los resultados obtenidos? No es fácil de encontrar una respuesta a la luz de la vasta evidencia sobre las ventajas de una condición robusta en el nacimiento. Sin embargo, cabe plantear la posibilidad de que, ante la vulnerabilidad de un hijo prematuro, la madre adapte su comportamiento y le proporcione cuidados compensatorios que redunden en un mejor desarrollo. Esta idea puede apoyarse en el peso que adquirieron las variables de crianza en los modelos de desarrollo.

Los datos muestran que los niños que reciben de sus cuidadores una dieta suficiente y diversa, 
puntúan mejor en las pruebas. Estos resultados van en el mismo sentido de los estudios antecedentes y fortalecen todos los supuestos sobre la relación entre una buena alimentación y el desarrollo infantil. De igual forma, los factores del CuPRE, relativos a la disposición a la interacción y la promoción de competencias, resultaron tener el efecto esperado sobre los puntajes del Bayley. No obstante lo anterior, los factores diseñados para valorar práctica responsiva y atención planeada mostraron una relación negativa sobre el desarrollo. Sería difícil afirmar, a partir de estos resultados, que las acciones de madres que están pendientes de las necesidades del niño tienen un efecto negativo sobre su desarrollo. Sin embargo, existen al menos dos explicaciones alternativas: la primera tiene que ver con la administración del instrumento y la eventualidad de que los cuidadores no respondieran en forma cuidadosa; la segunda se relaciona con el diseño del instrumento mismo y plantea la posibilidad de que estos factores estén dando información sobre estrés de crianza en lugar de responsividad de la práctica de crianza, y realmente refleje un estilo de atención del niño que afecta el desarrollo infantil, al alterar la dinámica de las interacciones, como lo plantea Ramsay (2004). Para estar en condiciones de una interpretación más sólida, es necesario aplicar el instrumento en condiciones diversas, para evaluar si efectivamente da cuenta de prácticas de crianza sensibles o este factor está indicando una conducta reactiva poco eficiente, en vez de responsividad.

Para finalizar, hay que subrayar que los modelos obtenidos muestran un nivel adecuado de explicación de la varianza, tanto para los indicadores antropomórficos como para los índices de desarrollo, señalando la conveniencia de considerar en el análisis de la desnutrición y sus efectos sobre el desarrollo infantil, la inclusión de variables ambientales de carácter distal y proximal. Este estudio aporta información que puede ser de valor para el desarrollo de intervenciones orientadas a prevenir la desnutrición infantil y atenuar sus efectos sobre el desarrollo.

\section{Referencias}

Ávila, A., Chávez, A., Shamah, T. \& Madrigal, H. (1993). La desnutrición infantil en el medio rural mexicano: análisis de las encuestas nacionales de alimentación. Salud Pública de México, 35 (6), 658-666.

Bayley, N. (1977). Escalas de Bayley de Desarrollo Infantil (BSID). Madrid: Técnicos Especialistas Aplicados. (Trabajo original publicado en 1969)

Black, M. (2003). Micronutrient deficiencies and cognitive functioning. Journal of Nutrition, 133 (Suppl. 2), 3927S-3931S.

Chávez, A., Martínez, H. \& Bourges, H. (1976). Nutrition and development of children from poor rural areas. En R. Pérez Hidalgo \& A. Chávez (Eds.), La desnutrición y la salud en México. México: Instituto Nacional de Nutrición, Publicación L-34.

Chopra, M. (2002). Risk factors for undernutrition of young children in a rural area of South Africa. Public Health Nutrition, 6 (7), 645-652.

Cortés, A., Romero, P. \& Flores, G. (2006). Diseño y validación inicial de un instrumento para evaluar prácticas de crianza en la infancia. Universitas Psychologica, 5 (1), 37-49.

Cravioto, J. \& Arrieta, R. (1982). Nutrición, desarrollo mental, conducta y aprendizaje. México: Instituto Nacional de Ciencias y Tecnología de la Salud del niño/DIF/UNICEF.

De Andraca, O., Salas, Y., De la Parra, A. \& González, B. (1993). Interacción madre-hijo y conducta del niño en preescolares con antecedentes de anemia por deficiencia de hierro en la infancia. Archivos Latinoamericanos de Nutrición, 43(3), 191-198.

Drewett, R., Wolke, D., Asefa, M., Kaba, M. \& Tessema, F. (2001). Malnutrition and mental development: Is there a sensitive period? A nested case-control study. Journal of Child Psychology and Psychiatry, 42 (2), 1881-1887.

Grantham-McGregor, S., Powell, C., Walker, S. \& Himes, J. (1991). Nutritional supplementation, psychosocial stimulation, and mental development of stunted children: The Jamaican study. Lancet, 338, 1-5.

Hernández, D., Barberena, C., Camacho, J. \& Vera, H. (2003). Desnutrición infantil y pobreza en México, 
Serie Cuadernos de Desarrollo Humano, 12. México: Secretaría de Desarrollo Social.

Hughes, D. \& Bryan, J. (2003). The assessment of cognitive performance in children: Considerations for detecting nutritional influences. Nutritional Reviews, 61 (12), 413-422.

Instituto Nacional de Ciencias Médicas y Nutrición Salvador Zubirán \& Sociedad Latinoamericana de Nutrición. (2003). Cambios en la situación nutricional de México de 1990 a 2000 a través de un Índice de Riesgo Nutricional por Municipio. México: Autor.

Kerr, M. A. \& Maureen, M. B. (2000). Failure-to-thrive, maltreatment and the behavior and development of 6-year-old children from low-income, urban families: A cumulative risk model. Child Abuse and Neglect, 24 (5), 587-598.

Kerr, M. A., Bogues, J. L. \& Kerr, D. S. (1978). Psychosocial functioning of mothers of malnourished children. Pediatrics, 62 (5), 778-784.

Krugman, S. D. \& Dubowitz, H. (2003). Failure to thrive. American Family Physician, 68 (5), 879-884.

Lamontagne, J. F., Engle, P. \& Zeitlin, M. F. (1997). Maternal employment, child care, and nutrition status of 12-18 month-old children in Managua, Nicaragua. Social Science \& Medicine, 46 (3), 403. 414.

Lima M. C., Eickmann S. H., Lima A. C. V., Guerra M. Q., Lira, P. I., Huttly S. R. et al. (2004). Determinants of mental and motor development at 12 months in a low income population: A cohort study in northeast Brazil. Acta Paediatrica, 93, 969-975.

Lozzoff, B., Klein, N., Nelson, L., McClish, D., Manuel, M. \& Chacon, E. (1998). Behavior of infants with iron-deficiency anemia. Child Development, 69 (1), 24-36.

Myers, R. (1993). Comprensión de las diferencias culturales en las distintas prácticas y creencias relativas a la crianza de los niños. En R. Myers, Los doce que sobreviven. Washington: Organización Panamericana de la Salud.

Parkinson, K. N., Wright, C. M. \& Drewett, R. F. (2004). Mealtime energy intake and feeding behaviour in children who fail to thrive: A population-based case-control study. Journal of Child Psychology and Psychiatry, 45 (5), 1030-1035.
Pelto, G. (2000). Improving complementary feeding and responsive parenting as a primary component of interventions to prevent malnutrition in infancy and early childhood. Pediatrics, 106, 1300-1301.

Peña, M. \& Bacallao, J. (2002). Malnutrition and poverty. Annual Review of Nutrition, 22, 241-254.

Poehlmann, J. \& Fiese, B. (2001). Parent-infant interaction as a mediator of the relation between neonatal risk status and 12-month cognitive development. Infant Behavior \& Development, 24, 171-188.

Pollitt, E., Golub, M., Grantham-McGregor, S., Levitsky, D., Schürch, B. et al. (1996). A reconceptualization of the effects of undernutrition on children's biological, psychological, and behavioral development (Social Policy Report). Society for Research in Child Development, 10 (5), 1-22.

Prentice, A. M., Gershwin, M. E., Schaible, U. E., Keusch, G. T., Victora, C. G. \& Gordon, J. I. (2008). New challenges in studying nutritiondisease interactions in developing world. The Journal of Clinical Investigation, 118 (4), 1322-1329.

Ramsay, M. (2004). Feeding skill, appetite and feeding behaviours of infants and young children and their impact on growth and psychosocial development. [Versión electrónica]. Encyclopedia on Early Childhood Development. Recuperado el 3 de marzo de 2009, de http://www .excellence-earlychildhood. ca/RamsayANGxp.pdf

Ribes, E. (1990). Psicología y salud: un análisis conceptual. Barcelona: Martínez Roca.

Ruel, M. T. \& Menon, P. (2002). Child feeding practices are associated with child nutrition status in Latin America: Innovative uses of the demographic and health surveys. Journal of Nutrition, 132, 1180-1187.

Sánchez-Sosa, J. J. (1998). Desde la prevención primaria hasta ayudar a bien morir: la interfaz, intervención-investigación en Psicología de la Salud. En G. Rodríguez \& M. Rojas (Coords.), La Psicología de la Salud en América Latina (pp. 33-44). México: Porrúa/UNAM.

Sazawal, S., Bentley, M., Balck, R., Dhingra, P., George, S. \& Bhan, M. (1996). Effect of zinc supplementation on observed activity in low socioeconomic Indian preschool children. Pediatrics, 98 (6), 1132-1137. 
Secretaría de Salud. (1999). Norma Oficial Mexicana para la atención a la salud del niño. Febrero 9 de 1999. Diario Oficial de la Federación NOM-031. SSA2-1999.

Seemba, R., De Pee, S., Sun, K., Sari, M., Akhter, N. \& Bloem, M. (2008). Effect of parental formal education on risk of child stunting in Indonesia and Bangladesh: A cross-sectional study. Lancet, 371, 322-328.

Velázquez, A., Larramendy, J. \& Rubio, J. (1998). Factores de riesgo de desnutrición proteico-energética en niños menores de 1 año de edad. Revista Cubana de Alimentación y Nutrición, 12 (2), 82-85.

Villalpando, S., Shamah, T., Ramírez, C. I., Mejía, F. $\&$ Rivera, J. A. (2003). Prevalencia de anemia en niños de 1 a 12 años de edad. Resultados de una encuesta probabilística nacional de México. Salud Pública de México, 45 (Supl. 4), S490-S498.
Wachs, T. D. (2000). Nutritional deficits and behavioural development. International Journal of Behavior Development, 24 (4), 435-441.

Walker, S., Wachs, T., Meeks Gardner, J., Lozoff, B., Wasserman, G., Pollitt, E. et al. (2007). Child development: Risk factors for adverse outcomes in developing countries. Lancet, 369, 145-157.

World Health Organization. (1995). Physical Status: The use and interpretation of anthropometry (Report of a WHO Expert Committee). Geneva, Switzerland: Author

World Health Organization. (2006). ANTHRO 2005: Software for assessing growth and development of the world's children [Computer software]. Geneva, Switzerland: Author.

Wright, C. \& Birks, E. (2000). Risk factors for failure to thrive: A population-based survey. Child, Care, Health and Development, 26 (1), 5-16. 